\title{
Caracterización morfológica del cerdo criollo (Sus scrofa domesticus) en el municipio de Nueva Guinea, RACCS
}

\section{Morphological characterization of local pig (Sus scrofa domesticus) in Nueva Guinea municipality, RACCS}

\author{
Marlon Hernández-Baca ${ }^{1}$, Alonso Gámez-Rivas², Yasser Zeledón-Ortega² \\ ${ }^{1} \mathrm{MSc}$ en Sistemas Integrales de producción agropecuaria en el trópico, Universidad Nacional Agraria / ${ }^{2}$ Ingeniero zootecnista
}

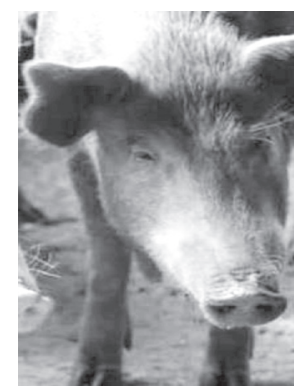

\section{RESUMEN}

El cerdo criollo es un recurso genético olvidado, que actualmente se encuentra sumamente amenazado por el mestizaje de su material genético con diferentes razas especializadas. Con el objetivo de realizar su caracterización morfométrica, faneróptica, la relación de variables con el sexo y la edad e identificación de biotipos, se realizó el presente estudio, evaluándose un total de 36 machos y 57 hembras en el municipio de Nueva Guinea. Se determinó la edad y 12 medidas morfológicas a partir de las cuales se calcularon 7 índices zoométricos: cefálico (ICF), proporcionalidad (IPD), corporal (ICP), profundidad relativa de pecho (IPRP), torácico (ITO), pelviano (IPV), metacarpo torácico (IMT), y las características fanerópticas en cada uno de los individuos. Del análisis estadístico descriptivo se observó que las medidas zoométricas evaluadas presentaron un rango de variación entre $13.24 \%$ (LK) y $30.68 \%$ (DDE), reflejando que se trata de individuos con una alta variabilidad morfoestructural. El análisis de regresión reveló que todas las medidas corporales están relacionadas significativamente con la edad, no así con la variable sexo que solo presento relación positiva con cinco variables y en el total de los casos no son significativas. Respecto a los índices zoométricos, estos cerdos pueden caracterizarse como dolicocéfalos ( $\mathrm{ICE}=42.51)$, longilíneos $(\mathrm{ICO}=98.75)$ y convexilíneos (IPE), con un tronco relativamente profundo e intermedio entre lo elíptico y lo cilíndrico, reflejando valores de índice torácico coincidente con su condición de longilíneos. En las características fanerópticas, se destaca que en estos cerdos predominan las capas y mucosas negras, cuerpo con abundante pelo, ausencia de mamelas y sindactilia, con predominio de perfiles rectos. Por el análisis clúster se clasificaron cuatro grupo de animales que presentaron diferencias significativas entre cada una de las variables morfométricas evaluadas. Los resultados obtenidos desprenden que se trata de individuos con alta variabilidad morfoestructural y por lo tanto es difícil hacer una tipificación zoométrica de la raza, y por otro lado que los grupos de individuos diferenciados por cluster, aun y cuando presentan diferencias estadísticamente significativas entre las distintas variables, no pueden ser considerados como biotipos diferenciados genéticamente, sino consecuencia del ambiente, necesitando de un tratamiento a nivel molecular y no simplemente basado en medidas zootécnicas.

Palabras clave: morfómetrica, fenotipico,bíotipos, índice zoometríco, morfoestructural.
Recibido: 22 de noviembre 2016 Aceptado: 19 de febrero del 2017
The creole pig is a forgotten genetic resource, which at the moment is extremely threatened by the mestization of its genetic material with different specialized races. The present study was carried out with a total of 36 males and 57 females in the municipality of Nueva Guinea with the objective of characterizing the morphometric, phaneroptic, relationship of variables with sex and age and identification of biotypes. Age and 12 morphological measures were determined, which were used to calculate 7 zoomometric indexes: cephalic (ICF), proportionality (IPD), body (ICP), chest depth (IPRP), thoracic (ITO), pelvic (IPV), Thoracic metacarp (IMT), and phaneroptic characteristics in each individual. From the descriptive statistical analysis it was observed that the zoomometric measures evaluated reached a range of variation between $13.24 \%$ (LK) and $30.68 \%$ (DDE), reflecting that they are individuals with a high morphostructural variability. The regression analysis revealed that all body measurements are significantly related to age, but not with the variable sex that only presented positive relation with five variables and in the total of the cases are not significant. With respect to zoomometric indexes, these pigs can be characterized as dolichocephalous $(\mathrm{ICE}=42.51)$, longilineal $(\mathrm{ICO}=98.75)$ and convexilineal (IPE), with a relatively deep trunk between the elliptic and cylindrical, reflecting values of coincident thoracic index With their condition of longils. In the phaneroptic characteristics, it is emphasized that these pigs predominate the layers and black mucous, body with abundant hair, absence of mamelles and syndactyly, with predominance of straight profiles. For the cluster analysis, four groups of animals were classified as having significant differences between each of the morphometric variables evaluated. The results obtained allow us to know that they are individuals with high morphostructural variability and therefore it is difficult to do a zoomometric typing of the race, and on the other hand that the groups of individuals differentiated by cluster, even though they present statistically significant differences between the Different variables, cannot be considered as genetically differentiated biotypes, but rather a consequence of the environment, necessitating a treatment at a molecular level and not simply based on zootechnical measures.
Aceptado: 19 de febrero del 2017 (c)
Los artículos de la revista La Calera de la Universidad Nacional Agraria, Nicaragua, se comparten bajo términos de la licencia Creative Commons: Reconocimiento, No Comercial, Compartir Igual. Las autorizaciones adicionales a las aquí delimitadas se pueden obtener en el correo freddy.aleman@ci.una.edu.ni 21 
$\mathrm{E}$ n las áreas rurales de la zona norte y de las regiones autónomas del atlántico norte y sur, aun sub- sisten sistemas de producción en los cuales es posible encontrar porcinos criollos, que son fieles sobrevivientes de los suinos traídos al país por los conquistadores españoles. Inicialmente introducidas por Pedrarias Dávila en el 1527, procedentes de Castillo de Oro, hoy Panamá y pertenecientes a su hacienda personal. Subsiguientes introducciones de razas ibéricas se dieron para garantizar la política de conquista de nuevos territorios y fundación de pueblos, posesionándose de los territorios descubiertos por el capitán Gil González Dávila en 1523. (Guerrero y Soriano 1992).

A lo largo de la historia los porcinos de origen ibérico se fueron consolidando en el tiempo en diferentes condiciones agroecológicas y constituyeron grupos genéticos denominados en su más simple expresión como razas criollas y por la FAO como recursos zoogenéticos, y cuyo uso está limitado comúnmente al auto consumo o ahorro familiar.

A inicios del siglo pasado, se realizan nuevas introducciones de razas porcinas, (Albarracín, B. M. 2014). Los nuevos genotipos, fueron absorbiendo y desplazando a las criollas, y a la par se produjo el desarrollo de nuevos sistemas de producción animal, que se generalizaron después de la segunda guerra mundial, debido a la industrialización del sector agropecuario. Llegada la Revolución verde, nuevos biotipos suinos de mayor potencial productivo y comercial, desplazaron a las primeras y a las segundas, sobresaliendo solo aquellas que podrían tener oportunidad en el mercado.

Pasados 490 años de adaptación de estos recursos zoogenéticos a las condiciones climáticas y la orientación productiva, que los campesinos le fueron dando, se formaron con características propias e integrales con la economía campesina en cada región del país, exhibiendo alta heterogeneidad, en comparación con las razas mejoradas.

Pocas investigaciones se han desarrollado para caracterizar estos suinos en vías de extinción y es por ello que el presente estudio pretende aportar elementos importantes de la morfometría y faneróptica del cerdo criollo, tratando de encontrar grupos de individuos con características comunes que pudiesen agruparlos en diferentes biotipos claramente definidos.

\section{MATERIALES Y MÉTODOS}

El presente estudio se realizó en el municipio del Nueva Guinea, Región Autónoma de la costa caribe Sur de Nicaragua, (RACCS), con coordenadas: $11^{\circ} 41^{\prime}$ latitud norte y $84^{\circ} 27^{\prime}$ longitud oeste, con temperaturas medias de $24.6^{\circ} \mathrm{C}$ y precipitaciones promedio anuales de 2245 milímetros (INETER 2017), lo cual la ubica según clasificación de Koeppen, en una zona de trópico húmedo.

La selección de las comarcas se realizó teniendo en cuenta la información primaria que se obtuvo de las instituciones que trabajan en la zona (INTA, MAG y Alcaldía), a lo que se agregó el criterio de accesibilidad de las comarcas, la información sobre mayor o menor números de cerdos criollos en las mismas, y la presencia de programas de asistencia técnica que facilitaran llegar de mejor manera a los productores de dichas comunidades. Las unidades de producción campesina se seleccionaron en base a cuatro condiciones básicas, tales como la disposición expresa de colaborar por parte del jefe de familia, la tenencia de diferentes categorías, desde lechones hasta cerdos adultos (seis meses a más), que no procedieran de cruces con razas de cerdos mejorados y que las hembras no estuvieran grávidas.

El número de visitas a los productores, dependió del tiempo que se tardó en la obtención de la información por finca, que estuvo en función del número de animales o categorías encontradas en cada una y el tiempo que tomaron las actividades de captura, medición y registro de los diferentes carácteres en los individuos encontrados. Para levantar la información de cada finca, se elaboró una encuesta estructurada que se aplicó por productor y por cada animal, en la cual se recogió la información general del productor, la información general de cada cerdo y las mediciones morfométricas y fanerópticas correspondientes.

En base a la metodología e indicaciones de Hurtado et al., (2004), 12 medidas morfométricas fueron tomadas, Longitud de la cabeza (LK), Longitud de cara (LC), Anchura de la cabeza (AK), Alzada a la cruz (AC), Alzada de la grupa. (AG), Diámetro longitudinal (DL), Diámetro dorso esternal (DDE), Diámetro bicostal (DB), Anchura de la grupa (ANG), Longitud de la grupa (LG), Perímetro torácico (PT) y Perímetro de la caña (PC). Estas medidas corporales fueron utilizadas para calcular 7 índices zoométricos, Índice cefálico (ICF), Indice de proporcionalidad (IPD), Índice corporal (ICP), Índice pelviano (IPV), Índice torácico (ITO), Profundidad relativa del pecho (IPRP) e Índice metacarpo torácico (IMT). Las características fanerópticas, seis en total, se registraron según las recomendaciones de Hurtado, (2004), Bermejo (2005), Revidatti, (2005) y barba, et al, (2011); las cuales fueron: Color de la capa (CC), Color de la mucosa (KM), Cobertura de pelo en el cuerpo (CP), Presencia de Mamelas (MM), Tipo de Perfil (TP) y Sindáctilia (CM).

En el análisis estadístico de la información se utilizó el software MINITAB 16, desarrollándose un análisis de estadística descriptiva, sobre todas las variables cuantitativas, determinando, media aritmética, desviación estándar y coeficiente de variación. Se desarrolló un análisis de regresión entre todas las variables o medidas morfológicas determinadas, teniendo como predictores el sexo y la edad. La ecuación de la recta de regresión de mínimos cuadrados a la que se ajustó el análisis fue:

$y=a+b x$

Donde:

$\mathrm{y}=$ Variable respuesta.

$\mathrm{a}=$ Valor de " $\mathrm{y}$ " cuando la variable predictora " $\mathrm{x}$ " es igual a cero.

$\mathrm{b}=$ Pendiente de la recta.

En la determinación de grupos con similitudes morfológicas, se desarrolló un análisis multivariado, utilizando la metodología Clúster y la técnica de Complete Linkage Method, con distancias cuadradas Euclidianas, estandarizando los datos de las medidas corporales, por tener estas diferentes unidades de medición. Las variables cualitativas recibieron 
un tratamiento especial, asignándoles valores numéricos, que permitieron introducirlas en el análisis de agrupamiento por aproximación de las diferentes características. Finalmente se realizó un análisis de varianza para determinar si las diferencias entre cada una de las variables de los animales ubicados en cada clúster eran o no significativas.

\section{RESULTADOS Y DISCUSIÓN}

Morfometría de los cerdos estudiados. En todas las variables morfométricas, los cerdos criollos estudiados, presentan una alta variabilidad morfoestructural, presentando coeficientes de variación muy altos que fluctuaron entre 13.24 $\%$ (longitud de la cabeza, LK) y 30.68\% (Diámetro dorso esternal, DDE). Según Roche et al (2006), los coeficientes de variación son muy relevantes en los estudios morfoestructurales, ya que cuando estos alcanzan valores entre 5 y $9 \%$, se debe pensar en comenzar a aplicar procesos de selección que contribuyan a disminuir la variabilidad de las diferentes regiones. En el cuadro 1, pueden observarse todos los valores obtenidos en las variables corporales y la edad que presento la más alta variabilidad.

Cuadro 1. Variables zoométricas de los cerdos criollos en el municipio de Nueva Guinea

\begin{tabular}{|c|c|c|c|c|c|}
\hline Variables & $\begin{array}{l}\text { exo } \\
36, \mathrm{H}=57)\end{array}$ & Media & D.ST & SE & C.V \\
\hline \multirow[t]{2}{*}{ Edad. } & $\mathrm{M}$ & 11.56 & 6.32 & 1.05 & 54.67 \\
\hline & $\mathrm{H}$ & 12.21 & 7.81 & 1.03 & 63.98 \\
\hline \multirow[t]{2}{*}{ Longitud de cabeza. (LK). } & M & 27.30 & 3.62 & 0.60 & 13.24 \\
\hline & $\mathrm{H}$ & 28.02 & 4.39 & 0.58 & 15.68 \\
\hline \multirow[t]{2}{*}{ Longitud de cara. (LC). } & M & 18.75 & 2.85 & 0.48 & 15.21 \\
\hline & $\mathrm{H}$ & 18.40 & 2.89 & 0.38 & 15.70 \\
\hline \multirow[t]{2}{*}{ Anchura de la cabeza (AK). } & M & 11.97 & 2.13 & 0.36 & 17.80 \\
\hline & $\mathrm{H}$ & 11.54 & 1.58 & 0.21 & 13.70 \\
\hline \multirow[t]{2}{*}{ Alzada de la cruz. (AC). } & M & 63.53 & 11.07 & 1.84 & 17.42 \\
\hline & $\mathrm{H}$ & 60.58 & 10.31 & 1.37 & 17.01 \\
\hline \multirow[t]{2}{*}{ Alzada de la grupa. (AG). } & M & 65.69 & 11.10 & 1.85 & 16.89 \\
\hline & $\mathrm{H}$ & 62.05 & 9.36 & 1.24 & 15.08 \\
\hline \multirow[t]{2}{*}{ Diámetro longitudinal (DL). } & M & 95.70 & 19.12 & 3.19 & 17.12 \\
\hline & $\mathrm{H}$ & 94.39 & 20.24 & 2.68 & 18.34 \\
\hline \multirow{2}{*}{ Diámetro dorso esternal (DDE). } & $\mathrm{M}$ & 35.89 & 9.82 & 1.64 & 27.36 \\
\hline & $\mathrm{H}$ & 36.70 & 11.26 & 1.49 & 30.68 \\
\hline \multirow{2}{*}{ Diámetro bicostal (DB). } & M & 26.11 & 4.82 & 2.39 & 18.47 \\
\hline & $\mathrm{H}$ & 28.91 & 5.08 & 1.79 & 17.60 \\
\hline \multirow[t]{2}{*}{ Anchura de la grupa (ANG). } & M & 17.25 & 3.52 & 0.59 & 20.43 \\
\hline & $\mathrm{H}$ & 19.18 & 3.28 & 0.43 & 20.27 \\
\hline \multirow{2}{*}{ Longitud de la grupa (LG). } & M & 20.40 & 7.49 & 1.25 & 16.90 \\
\hline & $\mathrm{H}$ & 21.50 & 10.81 & 1.43 & 23.56 \\
\hline \multirow[t]{2}{*}{ Perímetro toráxico (PT). } & M & 96.94 & 17.67 & 2.95 & 18.23 \\
\hline & $\mathrm{H}$ & 95.54 & 16.52 & 2.19 & 17.29 \\
\hline \multirow[t]{2}{*}{ Perímetro de la caña (PC) } & M & 17.97 & 2.44 & 0.41 & 13.60 \\
\hline & $\mathrm{H}$ & 17.13 & 2.40 & 0.32 & 14.02 \\
\hline
\end{tabular}

El promedio de edad en machos y hembras fue próximo al año de edad, lo que difiere de los encontrados por Lorenzo, M. (2012) quien obtuvo edades inferiores de 8 y 9 meses en cerdos criollos Guatemaltecos, con CV altos pero de menor valor a los nuestros.

En el cuadro 2, se puede observar la comparación de nuestros resultados con las medidas morfométricas reportadas por diferentes autores a nivel de Latinoamérica.

La longitud de la cabeza (LK), alcanzo mayor valor en las hembras, cuyos promedios de edades fueron mayores, ubicándose muy próximos a los reportados en el cerdo valencia, y en los criollos mexicanos, no así en los criollos de Guatemala con longitudes promedio iguales en ambos sexos.
En la longitud de la cara (LC), los valores fueron muy similares en machos y hembras, se aproximaron más a los reportados, en el cerdo cubano y en el cerdo mexicano y muy distante de lo reportado para el cerdo de Riobamba (Ecuador) y en cerdos guatemaltecos.

Para el ancho de la cabeza (AC), los valores no presentaron mayores diferencia en machos y hembras, en latinoamérica, los más próximos fueron los valores de los cerdos guatemaltecos, los del cantón de Riobamba y la Mana en ecuador e inferiores los valores reportados en cerdos venezolanos.

Los machos presentaron la mayor alzada a la cruz (AC), y se encontró similitud con la mayoría de los valores reportados en Latinoamérica, con excepción de las magnitudes reportadas para el cerdo criollo guatemalteco. Según Revidatti (2009), esta característica es poco influenciada por los factores ambientales, siendo un carácter estable en relación a las otras medidas corporales, y por ello se basan en ella la mayoría de los índices que se determinan, permitiendo comparar y diferenciar la génesis de los individuos.

En la alzada a la grupa (AG), los machos promediaron mayores valores, superando a los cerdos guatemaltecos, pero inferiores a las medidas reportadas para los cerdos, cubanos y ecuatorianos, (Valencia y La Mana). Caiza (1990), refiere que la alzada de la grupa es superior en los machos posiblemente debido a la adaptación que desde la etapa de crecimiento se viene diferenciado en ellos para una mayor facilidad en la monta natural. Por otro lado es importante observar que los valores de alzada a la cruz y alzada a la grupa son casi idénticos, los que nos da una idea que estos cerdos presentan una línea dorso-lumbar recta, bastante horizontal.

El ancho de la grupa (ANG), fue mayor en las hembras, las cuales solamente se diferenciaron de las reportadas para los cerdos ecuatorianos, de los cantones Valencia y La Mana que fueron superiores y los cerdos guatemaltecos con valores inferiores pero coincidentes en el hecho que dichas medidas sean mayores en las hembras.

Para los valores del largo de la grupa (LG), las hembras presentaron un ligero incremento, muy similar a los encontrados en los cerdos venezolanos e inferiores a los reportados para los cerdos ecuatorianos Chambo, Valencia y la Mana.

En relación al perímetro torácico $(\mathrm{PT})$, este fue mayor en los machos, muy similar a la mayoría de los estudios en Latinoamérica, con la excepción de los cerdos venezolanos y los guatemaltecos, cuyos valores fueron muy inferiores. Aunque el PT es la medida más influenciada por la alimentación, se corresponde exactamente con el tamaño y forma del tronco, y alcanza sus máximos valores en periodos tempranos del desarrollo del animal.

Las mediciones del perímetro de la caña (PC), fue prácticamente igual en machos y hembras, los cuales son superiores a la mayoría de los valores reportados en Latinoamérica, con la excepción de los cerdos venezolanos cuyos valores promedios son mayores.

En el diámetro longitudinal (DL), los machos presentaron una ligera ventaja, los cuales son similares a los reportados en los cerdos ecuatorianos por Falconi, C., \& Paredes, M. (2011), con medidas entre 95.46 y $73.37 \mathrm{~cm}$, pero 
muy diferentes a los señalados por Lorenzo, M. (2012) en los cerdos criollos guatemaltecos con magnitudes entre 53 y $23 \mathrm{~cm}$ para machos y hembras respectivamente. La alzada a la cruz y el diámetro longitudinal son medias necesarias para el conocimiento de las razas, siendo la segunda más variable, pero en ella influyen poco las condiciones de vida del animal, expresando su longitud.

El diámetro dorso esternal (DDE), resulto mayor en las hembras, teniendo proximidad con los reportadas en los cerdos ecuatorianos que alcanzaron valores entre $37.84 \mathrm{~cm}$ y $30.13 \mathrm{~cm}$, y superiores a los referidos para cerdos guatemaltecos que presentaron valores de $23 \mathrm{~cm}$ en los machos y 24 $\mathrm{cm}$ en las hembras.

El valor promedio del diámetro bicostal (DB), fue superior en las hembras, coincidente con los valores reportados en los cerdos ecuatorianos por Falconi, C y Paredes, M (2011) entre $28.02 \mathrm{~cm}$ y $23.06 \mathrm{~cm}$, reportándose valores inferiores en los cerdos guatemaltecos por Lorenzo, M. (2012), de $11 \mathrm{~cm}$ en machos y $12 \mathrm{~cm}$ en las hembras.

Cuadro 2. Medidas morfométricas en cerdos criollos latinoamericanos, reportadas por diferentes autores

\begin{tabular}{|c|c|c|c|c|c|c|c|c|c|c|}
\hline $\begin{array}{l}\text { Autor } \\
\text { estudio } \\
\text { (LK) }\end{array}$ & $\begin{array}{l}\text { Lugar de } \\
\text { cabeza } \\
\text { (L) }\end{array}$ & $\begin{array}{l}\text { Longitud } \\
\text { de cara } \\
\text { (LC) }\end{array}$ & $\begin{array}{l}\text { Longitud } \\
\text { de cabeza } \\
\text { (AK) }\end{array}$ & $\begin{array}{l}\text { Altura de } \\
\text { de cruz } \\
\text { (AC) }\end{array}$ & $\begin{array}{c}\text { Alza } \\
\text { de grupa } \\
\text { (AG) }\end{array}$ & $\begin{array}{c}\text { Alza } \\
\text { de grupa } \\
\text { (ANG) }\end{array}$ & $\begin{array}{r}\text { Ancho } \\
\text { grupa } \\
(\mathrm{LG})\end{array}$ & $\begin{array}{l}\text { Largo de } \\
\text { de tórax } \\
\text { (PT) }\end{array}$ & $\begin{array}{l}\text { Perímetro } \\
\text { de caña } \\
\text { (PC) }\end{array}$ & Perimetro \\
\hline $\begin{array}{l}\text { Yépez, } \\
2006 \\
\text { Ecuador }\end{array}$ & $\begin{array}{l}\text { Cerdo criollo } \\
\text { cantón } \\
\text { Riobamba }\end{array}$ & lo 29.8 & 26.5 & 12.4 & 60.5 & 61.6 & 19.6 & 18.2 & 93.2 & 14.8 \\
\hline $\begin{array}{l}\text { Escobar, } \\
2007\end{array}$ & $\begin{array}{l}\text { Cerdo criollo } \\
\text { cantón } \\
\text { Chambo }\end{array}$ & lo 29.9 & 15.8 & 16.0 & 63.3 & 62.9 & 20.2 & 28.7 & 96.6 & 15.9 \\
\hline $\begin{array}{l}\text { Estupiñán, } \\
\text { et al., } 2007\end{array}$ & $\begin{array}{l}\text { Cerdo } \\
\text { Valencia }\end{array}$ & 28.8 & 17.1 & 14.6 & 60.9 & 67.6 & 23.1 & 30.1 & 93.7 & 14.7 \\
\hline $\begin{array}{l}\text { Estupiñán, } \\
\text { et al., } 2007 \\
\text { Ecuador }\end{array}$ & $\begin{array}{l}\text { Cerdo } \\
\text { Naturalizado } \\
\text { La mana }\end{array}$ & 29.1 & 15.5 & 12.7 & 61.5 & 67.9 & 22.3 & 28.3 & 92.5 & 15.4 \\
\hline $\begin{array}{l}\text { Barba, } \\
\text { et al., } 1998\end{array}$ & $\begin{array}{l}\text { Cerdo } \\
\text { Cubano }\end{array}$ & 32.6 & 17.4 & 14.8 & 64.3 & 69.8 & 19.2 & 26.4 & 94.1 & 16.3 \\
\hline $\begin{array}{l}\text { Sierra, } \\
\text { et al., } 2005\end{array}$ & $\begin{array}{l}\text { Cerdo } \\
\text { Mexicano }\end{array}$ & 28.6 & 17.6 & & 60.8 & 65.9 & 19.9 & 26.8 & 91.0 & 13.5 \\
\hline $\begin{array}{l}\text { Hurtado, } \\
\text { et al., } 2004\end{array}$ & $\begin{array}{l}\text { Cerdo } \\
\text { Venezolano }\end{array}$ & 32.1 & 23.9 & 10.9 & 59.5 & 63.3 & 17.1 & 20.5 & 84.9 & 19.9 \\
\hline $\begin{array}{l}\text { Lorenzo } \\
\text { et al., } 2012\end{array}$ & $\begin{array}{l}\text { Cerdo } \\
\text { Chiquimula } \\
\text { Guatemala }\end{array}$ & 25.0 & 13.0 & 12.0 & 47.0 & 50.0 & 14.5 & 11.5 & 67.0 & 11.0 \\
\hline
\end{tabular}

ción entre $9.4 \%$ y $43.8 \%$, y se refieren a la proporción que de la variabilidad total en las medidas corporales en el modelo, son explicadas por la variable edad. En los valores inferiores al $40 \%$, resulta contradictorio el haber obtenido valores $(\mathrm{P}=0.000)$ significativos y comprobar por los resultados, que la edad no aporta una explicación muy grande de la variabilidad en la mayoría de las medidas corporales con excepción de las variable alzada a la grupa (AG) y diámetro longitudinal (DL) con valores de R2 de $43.6 \%$ y $43.8 \%$ respectivamente.

Relación entre las medidas corporales y el sexo. El análisis de regresión revelo, que aunque existen variaciones entre los valores de las variables corporales, según el sexo, tales diferencias no resultan significativas, siendo notorio en el cuadro 4, que los valores para las variables LK, DDE, DB, ANG, y $\mathrm{LG}$, presentaron valores positivos en la ecuación de la recta de regresión.

En relación a los coeficientes de determinación ajustados $\mathrm{R}^{2}$, en todas las medidas resulta con una fluctuación entre $0.00 \%$ y $1.8 \%$, y expresan cuanto de la variabilidad total de las medidas corporales puede ser explicada por la variable sexo. Estos valores concuerdan con la falta de significancia que el efecto del sexo tiene sobre la variación en las diferentes variables estudiadas y por tanto no era posible esperar que se prestaran valores altos en este coeficiente.

Valores de los índices zoométricos. Los 7 índices zoométricos, se estimaron a partir de las variables corporales presentadas en el cuadro uno, con la finalidad de determinar estados somáticos que permitieran derivar en determinadas funcionalidades y manifestaciones etnológicas, importantes en las definiciones particulares de las características raciales.
Relación entre las medidas corporales y la edad. Puede observarse en el cuadro 3, como todas las medidas corporales están relacionadas significativamente con la edad de los cerdos, esto dado por el hecho que en todas las ecuaciones de regresión, el efecto positivo de la edad sobre la variación en las medidas es notorio y puede leerse como el incremento en centímetros que estas tienen por cada mes que incrementan las edades.

Los valores extremos de los errores estándar encontrados fueron de 0.60 para longitud de la grupa (LG) y 14.77 para el diámetro longitudinal (DL), explicando dichos errores, los promedios en que los valores encontrados se alejan de las medidas reales de cada variable.

En relación a los coeficientes de determinación ajustados R2, en todas las medidas resulta con una fluctua-
Cuadro 3. Significancia de la regresión entre las medidas corporales y la edad

\begin{tabular}{lcrrr}
\hline Variable & $\begin{array}{l}\text { variación }(\mathrm{cm}) \\
\text { en relación a la edad }\end{array}$ & $\begin{array}{l}\mathrm{R} 2(\mathrm{adj}) \\
\%\end{array}$ & $\mathrm{~S}$ & $\mathrm{P}=0.05$ \\
\hline LK & 0.317 & 30.5 & 3.42 & 0.000 \\
LC & 0.23 & 33.2 & 2.34 & 0.000 \\
AK & 0.094 & 13.0 & 1.69 & 0.000 \\
AC & 0.79 & 28.6 & 8.96 & 0.000 \\
AG & 0.92 & 43.6 & 7.52 & 0.000 \\
DL & 1.82 & 43.8 & 14.77 & 0.000 \\
DDE & 0.72 & 23.1 & 9.36 & 0.000 \\
DB & 1.14 & 35.4 & 11.06 & 0.000 \\
ANG & 0.15 & 9.4 & 3.23 & 0.000 \\
LG & 0.615 & 20.4 & 0.60 & 0.000 \\
PT & 1.42 & 36.5 & 13.45 & 0.000 \\
PC & 0.177 & 26.7 & 2.08 & 0.000 \\
\hline
\end{tabular}


Cuadro 4. Significancia de la regresión entre las medidas corporales y sexo

\begin{tabular}{lccrc}
\hline Variable & $\begin{array}{l}\text { variación }(\mathrm{cm}) \\
\text { en relación al sexo }\end{array}$ & $\begin{array}{l}\text { R2 }(\mathrm{adj}) \\
\%\end{array}$ & $\mathrm{~S}$ & $\mathrm{P}=0.05$ \\
\hline LK & 0.71 & 0.00 & 4.11 & 0.418 \\
LC & -0.346 & 0.00 & 2.87 & 0.573 \\
AK & -0.428 & 0.30 & 1.81 & 0.270 \\
AC & -2.946 & 0.10 & 10.60 & 0.309 \\
AG & -3.64 & 0.00 & 10.06 & 0.765 \\
DL & -1.28 & 0.00 & 19.81 & 0.762 \\
DDE & 0.806 & 0.00 & 10.73 & 0.797 \\
DB & 2.75 & 0.00 & 13.83 & 0.835 \\
ANG & 1.89 & 1.30 & 3.37 & 0.138 \\
LG & 1.20 & 0.00 & 9.66 & 0.442 \\
PT & -1.40 & 0.00 & 16.97 & 0.699 \\
PC & -0.841 & 1.80 & 2.42 & 0.106 \\
\hline
\end{tabular}

En el cuadro 5, se pueden observar los valores promedio y diferenciados por sexo de los diferentes índices. El índice cefálico, principal índice zoométrico de diagnosis racial, alcanzo un valor promedio de 42.51 , con lo cual se pueden clasificar a los animales de esta población como dolicocéfalos, ya que en ellos predomina el largo de la cabeza por sobre el ancho. Herrera y Luque (2009), señalan que los caracteres étnicos referidos por la cabeza, como el índice cefálico, tienen su importancia etnológica, sobretodo porque su variación no está influenciada por los factores ambientales y el manejo.

Cuadro 5. Índices zoométricos diferenciados por sexo del cerdo criollo en el municipio de Nueva guinea

\begin{tabular}{lccc}
\hline Índice & Macho & Hembra & Media \\
\hline Índice cefálico. (ICF) & 43.85 & 41.18 & 42.51 \\
Índice de proporcionalidad. (IPD) & 66.38 & 64.18 & 65.28 \\
Índice corporal. (ICP) & 98.72 & 98.79 & 98.75 \\
Profundidad relativa de pecho. (IPRP) & 56.49 & 60.58 & 58.53 \\
Índice torácico. (ITO) & 72.75 & 78.77 & 75.76 \\
Índice pelviano. (IPV) & 84.55 & 89.20 & 86.87 \\
Índice metacarpo torácico. (IMT) & 18.54 & 17.92 & 18.23 \\
\hline
\end{tabular}

El índice de proporcionalidad resulto con un valor promedio de 65.28, este expresa la relación entre la alzada a la cruz y el diámetro longitudinal, delimitando la rectangularidad del cuerpo del animal. La interpretación de este índice requiere de un adecuado nivel de intuición puesto que a medida que el valor se aleja del $100 \%$ los animales serán más rectangulares, algunos autores consideran que es mejor si excede de $50 \%$, en todo caso los animales de nuestro estudio es claro que presentan formas rectangulares, aproximándose más a la actitud cárnica.

El índice corporal arrojo un promedio de 98.75, con lo cual se puede clasificar a estos animales como longilíneos, con cuerpos largos y relativamente anchos.

La profundidad relativa de pecho, alcanzo un promedio poblacional de 58.53, que puede considerarse como buena, puesto que se será mejor cuanto más exceda de 50 , debido a que los animales tendrán un tronco más profundo.

En los valores de índice torácico, el promedio general fue de 75.76, el cual se ubica adecuadamente para los animales longilíneos cuyos valores deben ser inferiores o igua- les a 85. Estos animales presentaron un tronco ligeramente más alto que ancho (35.89 a 26.11) y por esta relación de sus variables la forma del tórax presenta un estado intermedio entre lo elíptico y lo cilíndrico.

El índice pelviano alcanzó u valor promedio de 86.87. Este índice indica la relación entre anchura y longitud de pelvis, lo que refleja en nuestro caso una pelvis ligeramente más larga que ancha y clasificarse como convexilínea al tener un valor inferior a 100. Siendo importante tener en cuenta que a medida que el largo de la grupa se incrementa sobre el ancho, el valor del índice decrece.

En relación al índice metacarpo torácico (Dáctilo-Torácico), este alcanzo un promedio poblacional de 18.23, con lo cual puede clasificarse como alto, al superar un valor de 11. Este índice relaciona el perímetro de los metacarpos con la compacidad del cuerpo, indicando si son proporcionales, dándonos también una idea del grado de finura del esqueleto y si se favorecerá el sostén de las estructuras corporales. En nuestro caso el perímetro torácico es 5.5 veces el perímetro de la caña.

Las variables fanerópticas identificadas. El color de la capa en los cerdos de nuestro estudio, puede observarse en la cuadro 6 , que predominan los colores negro y rosado, con menores proporciones para las tonalidades blanco y manchados, Barba et al., 1998, en el cerdo cubano y Lorenzo, et al., (2012), en el cerdo guatemalteco coinciden en que las capas negras son predominantes aunque reportan diferentes proporciones.

En relación a la coloración de las mucosas, predomino la tonalidad rosada, seguida de las de aspecto negro y en menores proporciones las tonalidades manchadas y blancas. Estos resultados difieren de los reportados por otros autores en Latinoamérica Barba Capote, et al, 1998; Falconi, y Paredes, (2011), en los que las mucosas obscuras son las que predominan.

El mayor número de animales, presento pelo abundante, seguido de los que presentaron poco pelo y escasos 4 animales resultaron cerdos pelones. Estos resultados coinciden con Lorenzo, et al., (2012), en los cerdos criollos de Guatemala y Falconi y Paredes, (2011), en los cerdos ecuatorianos con el predominio de animales de pelo abundante en el cuerpo.

Cuadro 6. Frecuencias de características fanerópticas

\begin{tabular}{|c|c|c|c|}
\hline Carácter & & Valor absoluto & Frecuencia relativa $\%$ \\
\hline \multirow[t]{4}{*}{ Color de la capa } & Negro. & 40 & 43.01 \\
\hline & Rosado. & 37 & 39.78 \\
\hline & Blanco. & 7 & 7.53 \\
\hline & Manchado. & 9 & 9.68 \\
\hline \multirow{4}{*}{ Color de mucosas } & Blanco. & 3 & 3.23 \\
\hline & Manchado. & 8 & 8.60 \\
\hline & Negro. & 33 & 35.48 \\
\hline & Rosado. & 49 & 52.69 \\
\hline \multirow[t]{3}{*}{ Pelo en el cuerpo } & Abundante. & 73 & 78.5 \\
\hline & Poco pelo. & 16 & 17.20 \\
\hline & Pelón. & 4 & 4.30 \\
\hline \multirow{2}{*}{ Mamelas } & Ausencia. & 85 & 91.40 \\
\hline & Presencia. & 8 & 8.60 \\
\hline \multirow[t]{2}{*}{ Sindactilia } & Ausente. & 93 & 100 \\
\hline & Existente. & 00 & 00 \\
\hline \multirow[t]{3}{*}{ Tipo de perfil } & Cóncavo. & 14 & 15.00 \\
\hline & Sub-cóncavo. & 19 & 20.43 \\
\hline & Recto. & 60 & 64.52 \\
\hline
\end{tabular}


La mayor parte de los individuos muestreados no presentaron mamelas, por lo que la existencia de tal característica se reduce a pocos individuos y es coincidente con otros autores en latinoamérica como Lorenzo, et al., (2012), Revidatti, et al., (2004), en cerdos argentinos y Falconi y Paredes, (2011), asegurando estos últimos que es una característica de los cerdos ibéricos, que en los cerdos ecuatorianos se está perdiendo.

La Sindactilia (Cerdos casco de mula) que es una característica que se produce por fusión de la $2^{\mathrm{a}}$ y $3^{\mathrm{a}}$ falange de los dedos para crear una pezuña fusionada, estuvo ausente en los animales estudiados. Solamente Revidatti, et al., (2004), señala un cierto número de cerdos del nordeste argentino con pezuñas enteras (no hendidas), al igual que Lemus, et al., (2003) en cerdos nativos mexicanos, y se afirma que se trata de una ventaja, ya que estos cerdos son resistentes a la peste porcina y padecen menos problemas locomotores.

En los suinos estudiados predominaron los perfiles rectos, seguidos de los perfiles sub-cóncavos y los cóncavos, coincidiendo en el predominio de los perfiles rectos con Lorenzo, et al., (2012), en cerdos guatemaltecos y no así con Revidatti, et al., (2004), que reporto el predominio de los perfiles concavilíneos en cerdos criollos argentinos.

Polimorfismo de los cerdos criollos estudiados. A través del análisis cluster, considerando todas las variables corporales, se lograron establecer cuatro grupos con diferente número de individuos por cada uno, se realizó un análisis de varianza entre los cluster para cada variable y los resultados pueden observarse en el cuadro 7, en el cual es notorio que todas las medidas zoométricas resultan con diferencias significativas $(\mathrm{p}=0.05)$ entre cluster. Sin embargo, a pesar de estas diferencias estadísticas, no se puede afirmar que se trata de biotipos específicos diferenciados, sino más bien, que tales diferencias pueden deberse a factores ambientales de manejo y alimentación; tal afirmación puede reforzarse si tenemos en cuenta que las principales variables fanerópticas se encontraron distribuidas en todos los grupos de animales que conformaron cada cluster y que por lo tanto tampoco permitió hacer una diferenciación fenotípica particular para cada grupo de animales.

\section{CONCLUSIONES}

Los cerdos criollos de nuestro estudio presentan una alta variabilida en su condición morfoestructural, ya que el $100 \%$ de las variables corporales estudiadas tienen un coeficiente de variación mayor al 10\%, y por lo tanto es difícil hacer una tipificación zoométrica de la raza.

Todas las medidas corporales están influenciadas positivamente por la edad, pero únicamente dos variables $(\mathrm{AG})$ y (DL), reciben una alta explicación por la variable independiente, según el coeficiente de determinación ajustado $\mathrm{R}^{2}$.

Únicamente para cinco variables, el sexo tuvo una relación positiva. Sin embargo por el ANDEVA, se descarta que este tenga efectos significativos sobre la variabilidad de las distintas mediciones corporales.

Los índices zoométricos indican que se trata de una población de cerdos dolicocéfalos, longilíneos y convexilíneos, con un tronco relativamente profundo e intermedio entre lo elíptico y lo cilíndrico, reflejando valores de índice torácico coincidente con su condición de longilíneos.

Es una población de cerdos en los que predominan los colores de capas y mucosas Negras y Rosadas, con coberturas abundantes de pelo, desprovistos de mamellas y Sindactilia, con perfiles rectos en su gran mayoría.

A la vista de los resultados obtenidos en los diferentes clusters, podemos afirmar que, para los animales y comarcas con los que se trabajó, no podemos definir estos grupos de cerdos como biotipos distintos, siendo la variabilidad existente fruto de las diferencias en edades, así como por condiciones ambientales particulares en cada caso, fundamentalmente de la disponibilidad de alimentos.

Cuadro 7. Análisis de variable por cluster y variables morfométricas

\begin{tabular}{|c|c|c|c|c|c|c|c|c|c|c|c|c|c|}
\hline \multirow[t]{2}{*}{ Variable } & \multicolumn{3}{|c|}{ Cluster 1} & \multicolumn{2}{|c|}{ Cluster 2} & \multicolumn{4}{|c|}{ Cluster 3} & \multicolumn{2}{|c|}{ Cluster 4} & \multicolumn{2}{|c|}{$p=0.05$} \\
\hline & $X$ & StDV & $\mathrm{CV}$ & $X$ & StDV & $\mathrm{CV}$ & $X$ & StDV & $\mathrm{CV}$ & $\mathrm{X}$ & StDV & $\mathrm{CV}$ & \\
\hline LK & 27.59 & 4.18 & 15.15 & 26.38 & 5.22 & 19.79 & 30.50 & 4.63 & 15.19 & 28.36 & 5.26 & 18.54 & 0.009 \\
\hline $\mathrm{LC}$ & 18.53 & 2.42 & 13.07 & 17.65 & 1.99 & 11.27 & 20.13 & 3.58 & 17.78 & 19.00 & 4.24 & 22.33 & 0.034 \\
\hline $\mathrm{AK}$ & 11.62 & 1.41 & 12.13 & 10.97 & 1.99 & 18.14 & 13.31 & 2.75 & 20.66 & 11.90 & 2.02 & 16.97 & 0.000 \\
\hline $\mathrm{AC}$ & 59.64 & 13.76 & 23.07 & 60.75 & 15.05 & 24.77 & 63.00 & 11.27 & 17.88 & 65.00 & 14.44 & 22.21 & 0.000 \\
\hline $\mathrm{AG}$ & 62.78 & 7.081 & 11.28 & 64.05 & 7.61 & 11.88 & 65.63 & 10.34 & 17.75 & 67.64 & 12.88 & 19.04 & 0.000 \\
\hline DL & 87.34 & 15.25 & 17.46 & 90.89 & 16.48 & 18.13 & 100.45 & 22.9 & 22.79 & 98.56 & 20.18 & 20.47 & 0.000 \\
\hline DDE & 36.38 & 11.27 & 30.97 & 28.73 & 7.89 & 23.46 & 32.63 & 7.80 & 23.90 & 32.21 & 11.77 & 36.54 & 0.001 \\
\hline DB & 25.75 & 6.053 & 23.51 & 28.89 & 7.66 & 26.53 & 24.00 & 5.88 & 24.53 & 29.09 & 10.36 & 35.64 & 0.000 \\
\hline ANG & 18.76 & 3.20 & 17.05 & 16.31 & 2.09 & 12.81 & 20.25 & 4.55 & 22.46 & 19.72 & 3.20 & 16.23 & 0.001 \\
\hline LG & 15.64 & 3.00 & 19.18 & 19.92 & 3.02 & 15.16 & 21.75 & 5.53 & 25.42 & 23.64 & 5.76 & 24.36 & 0.002 \\
\hline PT & 91.13 & 10.74 & 11.78 & 87.71 & 15.52 & 17.69 & 110.56 & 15.39 & 13.92 & 100.64 & 21.85 & 21.71 & 0.000 \\
\hline PC & 17.05 & 2.17 & 12.72 & 16.53 & 1.67 & 10.10 & 19.75 & 3.07 & 15.54 & 18.18 & 2.09 & 11.49 & 0.000 \\
\hline
\end{tabular}




\section{REFERENCIAS BIBLIOGRÁFICAS}

Albarracín, BM. 2014. La Conservación del cerdo criollo (sus scrofa domestica), recurso alimentario de sistemas tradicionales de producción campesina. Pontificia universidad javeriana. 2014.

Benítez, OW; Sánchez, M. 2000. Los Cerdos locales en los sistemas tradicionales de producción. Estudio FAO, Producción y sanidad animal. $\mathrm{N}^{\circ} 148$.

Barba-Capote, CJ; Velázquez-Rodríguez, F; Perez-Freeman, F; Delgado Bermejo, JV. 1998. Contribución al estudio racial del cerdo Criollo cubano. Revista Archivos de Zootecnia, 47:51-59.

Bermejo, J.V.D; Prieto, PN; Capellari, A; Revidatti, MA. 2005. Estudio morfoestructural preliminar de una población porcina en la provincia de Corrientes. Argentina. Archivos de zootecnia, 54(206), 227-232.

Escobar, R, JC. 2007. Caracterización y Sistemas de Producción de los Cerdos Criollos del Cantón Chambo. Escuela Superior Politécnica Chimborazo. Ecuador.

Estupiñán, V; Vasco, D; Barreto, S; Zambrano, K. 2007. Estudio morfoestructural de una población de cerdos naturalizados en el cantón Valencia, provincia de Los Ríos, Ecuador. Memorias del VIII Simposio Iberoamericano sobre conservación y utilización de recursos Zoogenéticos. p 134-142.

Estupiñán, V; Vasco, D; Barreto, S; Zambrano, K. 2007. Estudio morfoestructural de una población de cerdos naturalizados en el cantón La Maná, provincia de Cotopáxi, Ecuador. Memorias del VIII Simposio Iberoamericano sobre conservación y utilización de recursos Zoogenéticos. p 166-172.

Falconí Velasco, CR; Paredes Barros, MX. 2011. Levantamiento poblacional, caracterización fenotípica y de los sistemas de producción de los cerdos criollos en los cantones de Mejía (Pichincha) y Colta (Chimborazo).

Guerrero y Soriano (1992). Historia de la Ganadería Nicaragüense. Edit. Unión. Managua. NI.

Herrera, M; Luque, M. (2009). Morfoestructura y sistemas para el futuro en la valoración morfológica. En: Valoración Morfológica de los animales domésticos. Sañudo, A, C. (Ed). Madrid, Ministerio de Medio Ambiente y Medio Rural y Marino.

Hurtado, E., González, C; Ly, J; No, GP; Brava, P. 2004. Estudio morfológico del cerdo criollo del Estado Apure, Venezuela. Revista Computarizada de Producción Porcina 11 (3).

Lorenzo, M; Jáuregui, J, Vásquez, Ch. 2012. Caracterización del Cerdo Criollo de la Región de Cho'rti' del departamento de Chiquimula, Guatemala. Centro Universitario de Oriente, Universidad de San Carlos. Guatemala. Actas Iberoamericanas de Conservación Animal. AICA 2 (2012) 103 103-108.

Revidatti, M. (2009). Caracterización de cerdos criollos del nordeste argentino. Universidad de Córdoba.

Roche, A; Salinas, M; Santander, L; Andaluz, E, Peña, F; Herrera, M. 2006. Raza Roya Bilbilitana. Caracteres cuantitativos morfoestructurales. In XXXI Jornadas científicas. Zamora. España.

Sierra, AC; Poot, TB; Díaz, ZI; Cordero, AH; Delgado, JV. 2005. El Cerdo Pelón Mexicano, una raza en peligro.

Yépez. R. 2006. Caracterización de los porcinos criollos mestizos en la comunidad de Pungala Escuela Superior Politécnica Chimborazo. Riobamba. Ecuador. 